\title{
Warfarin and Rivaroxaban Duplication: A Case Report and Medication Error Analysis
}

\author{
Julie A. Fusco ${ }^{1} \cdot$ Eric J. Paulus ${ }^{2} \cdot$ Alexandra R. Shubat $^{3} \cdot$ Sharminara Miah $^{3}$
}

Published online: 9 June 2015

(c) The Author(s) 2015. This article is published with open access at Springerlink.com

\begin{abstract}
A 62-year-old African American man received unintentional duplicate anticoagulation therapy with warfarin $5 \mathrm{mg}$ and rivaroxaban $20 \mathrm{mg}$ daily for the treatment of recurrent pulmonary embolism. The patient presented to the anticoagulation clinic 6 days after hospital discharge with an International Normalized Ratio (INR) of 2.3 and he was instructed to continue warfarin $5 \mathrm{mg}$ daily. Seven days later, he returned to the clinic with an INR $>8.0$ using a point-of-care device. He denied any signs or symptoms of bleeding. During the interview, he reported starting a new medication for neuropathy 5 days earlier. The clinical pharmacist contacted the dispensing pharmacy and determined rivaroxaban $20 \mathrm{mg}$ was the new medication. The patient denied receiving new prescription counseling at the dispensing pharmacy. Because rivaroxaban can falsely elevate INR results, the actual INR value was unknown. To minimize the risk for recurrent venous thromboembolism, vitamin $\mathrm{K}$ was not administered and no warfarin doses were held. Rather, the patient was instructed to stop rivaroxaban and reduce the warfarin dose. Five days later, the patient returned with an INR of 4.3. He still had not experienced any signs or symptoms of bleeding. The patient was quickly stabilized on a warfarin maintenance dose of $22.5 \mathrm{mg}$ weekly. The anticoagulation clinic pharmacist notified management at the clinic and at the
\end{abstract}

Julie A. Fusco

jannfusco@gmail.com

1 Department of Pharmacy Practice, Midwestern University Chicago College of Pharmacy, 555 31st Street, Downers Grove, IL 60515, USA

2 Advocate Medical Group, Chicago, IL, USA

3 Midwestern University Chicago College of Pharmacy, Downers Grove, IL, USA dispensing pharmacy in an effort to identify process errors and prevent additional incidents.

\section{Key Points}

Both warfarin and rivaroxaban are indicated for the treatment of pulmonary embolism.

Anticoagulant medications are one of the most common classes of medications associated with adverse effects and emergency room visits.

Poor communication between healthcare professionals and patients can increase the risk for adverse effects from medication therapy.

\section{Introduction}

Venous thromboembolism (VTE) affects an estimated 900,000 persons annually in the USA [1]. Comprised of deep venous thrombosis and pulmonary embolism (PE), the disease represents a significant health burden. For patients experiencing a thrombotic event, the 30-day mortality rate is $30 \%$ [2]. Warfarin is an effective traditional anticoagulant; however, a narrow therapeutic window necessitates laboratory monitoring of the International Normalized Ratio (INR) [3]. One study found that $17 \%$ of emergency department visits for adverse drug events (ADEs) among older adults were related to warfarin [4]. Numerous medication interactions with warfarin may be responsible for associated ADEs, including bleeding [5]. 
Because of the drawbacks of warfarin, medications that directly inhibit either coagulation factor II (thrombin) or factor Xa have more recently been developed. These anticoagulants are referred to as non-vitamin $\mathrm{K}$ oral anticoagulants (NOACs), and three of these agents (apixaban, dabigatran etexilate, and rivaroxaban) are currently approved for the treatment and prevention of VTE in the USA. NOACs have a faster onset, shorter half-life, as well as fewer medication and dietary interactions. Laboratory monitoring is unnecessary because the dose response is more predictable. However, they are more expensive and a specific antidote does not exist [2, 3]. In clinical practice, patients are increasingly inquiring about switching from warfarin to NOACs. During the transition, the potential for an ADE exists, such as recurrent VTE due to inadequate anticoagulation or bleeding due to therapeutic duplication. Classifying an ADE as potentially due to a medication error suggests process improvements can be made.

\section{Case Presentation}

A 62-year-old African American man was referred to a pharmacist-managed anticoagulation clinic for follow-up status post-extensive bilateral PE. The patient had received warfarin $10 \mathrm{mg}$ daily for 2 days during the hospitalization, then was discharged with $5 \mathrm{mg}$ daily. Six days after discharge, the patient presented for an initial anticoagulation clinic visit with an INR of 2.3 (goal INR 2.0-3.0). He was instructed to continue $5 \mathrm{mg}$ daily and to return 7 days later. At the next visit, point-of-care testing revealed an INR of $>8.0$. His last dose was taken in the morning the same day. The patient denied extra warfarin doses. There was no suggestion of concurrent acute illness or recent alcohol intake. He initially denied any new prescription or supplemental medications, and stated adherence with his maintenance medications, including chlorthalidone and losartan. He denied signs and symptoms consistent with bruising and bleeding. His active problem list included hypertension, osteoarthritis, sleep apnea, and gout. Past medical history included a PE less than 3 years earlier. At that time, he was maintained on warfarin $22.5 \mathrm{mg}$ weekly for a treatment duration of 18 months. Laboratory examination prior to hospital discharge revealed hemoglobin $11.1 \mathrm{~g} / \mathrm{dL}$, hematocrit $33.9 \%$, and platelets $134 \mathrm{~K} / \mathrm{mcL}$. Liver function tests were within the normal range. The creatinine level was $1.75 \mathrm{mg} / \mathrm{dL}$ with an estimated creatinine clearance of $46 \mathrm{~mL} /$ min (using ideal body weight).

On further questioning, the patient reported starting a new prescription medication 5 days earlier. The tablet was described as small and triangular shaped. The anticoagulation clinic contacted the patient's community pharmacy and determined the medication was rivaroxaban $20 \mathrm{mg}$. Communication among the anticoagulation clinic staff revealed that this prescription had been placed after the initial visit because the patient inquired about the cost of rivaroxaban with his insurance plan. The community pharmacy placed the rivaroxaban prescription on hold rather than discontinue the order as the anticoagulation clinic staff had requested. When the patient presented to his pharmacy the next day to pick up a different medication refill, the rivaroxaban prescription had been filled and was included in the orders ready for pick up. He reportedly did not receive counseling when it was dispensed. He thought it was a new medication for neuropathy, as this problem had been recently discussed with his primary care physician. Despite reading the term 'blood thinner' in the medication guide, the patient did not clarify its indication with the dispensing pharmacist or anticoagulation clinic. He had picked up the original warfarin prescription 8 days earlier.

The patient had a prolonged coagulation time; however, the actual INR was uncertain given the limitations of the point-ofcare device and the patient's reluctance to visit the laboratory for a venous blood draw. One consideration was the initial warfarin dosing was excessive, based on the comparison of his current dosing (5 $\mathrm{mg}$ daily) with his previous dose requirements ( $22.5 \mathrm{mg}$ weekly). There was also the possibility the coagulopathy was due to concurrent administration of rivaroxaban. When switching from warfarin to rivaroxaban, it is recommended to discontinue warfarin and start rivaroxaban when the INR is $<3.0$. The intent is to not only avoid inadequate anticoagulant effects during the transition but also to avoid duplication for an extended time interval [6].

The anticoagulation clinic considered several ways to manage this patient. The patient did not desire to continue rivaroxaban, so this alternative was dismissed. The administration of vitamin $\mathrm{K}$ as a reversal strategy for warfarin was one option but could have placed the patient at high risk for recurrent VTE. For this same concern, the staff were reluctant to hold warfarin doses with the possibility of a resultant subtherapeutic INR. The patient was ultimately advised to take warfarin $2.5 \mathrm{mg}$ daily for 3 days and then $5 \mathrm{mg}$ for 1 day. He was counseled to monitor for signs and symptoms of bleeding and to seek emergent care if symptomatic. At a morning visit 5 days later, the INR was 4.3. He denied signs and symptoms of bleeding. INR monitoring occurred weekly to biweekly over the next 6 weeks. A total weekly dose of $22.5 \mathrm{mg}$ was found to be sufficient to maintain INR in the goal range.

\section{Discussion}

\section{Rivaroxaban and INR Effect}

A brief review of rivaroxaban pharmacokinetics is useful for application to the case. Rivaroxaban reaches maximum (peak) plasma concentrations 2-4 h after oral 
administration [6]. Taken with food, time to peak plasma concentration increases from $2.5 \mathrm{~h}$ (after fasting) to $4 \mathrm{~h}$ [7]. Maximal factor Xa inhibition and maximal effect on prothrombin time (PT) are found approximately $2 \mathrm{~h}$ after ingestion [8]. Rivaroxaban has a terminal elimination halflife of 5-9 $\mathrm{h}$ in healthy young subjects (age 20-45 years), and 11-13 $\mathrm{h}$ in elderly subjects [6]. In patients with mild (creatinine clearance $50-80 \mathrm{~mL} / \mathrm{min}$ ) and moderate (creatinine clearance $30-49 \mathrm{~mL} / \mathrm{min}$ ) renal function, plasma concentrations were increased 1.4- and 1.5-fold, respectively [9]. Regarding patient weight, no relevant changes in pharmacokinetics and pharmacodynamics were observed at higher body weights $(>120 \mathrm{~kg})$, while moderately increased effects occurred at lower body weights $(<50 \mathrm{~kg})$ [10].

In a recent study of healthy male patients $(n=96)$, the mean maximum PT prolongation was 4.4-fold (range 3.4- to 6.5-fold) for patients being transitioned from warfarin (goal INR 2.0-3.0) to rivaroxaban $20 \mathrm{mg}$ once daily [11]. In comparison, monotherapy with rivaroxaban only produced a 1.6-fold (range 1.4- to 2.1-fold) mean maximum PT prolongation. Peak plasma concentrations after rivaroxaban $20 \mathrm{mg}$ were $223 \mathrm{ng} / \mathrm{mL}(160-360 \mathrm{ng} / \mathrm{mL})$, with trough levels $22 \mathrm{ng} / \mathrm{mL}(1-38 \mathrm{ng} / \mathrm{mL})$ [12]. The estimated maximum plasma concentration of rivaroxaban $20 \mathrm{mg}$ for DVT treatment is $270 \mathrm{ng} / \mathrm{mL}(189-419 \mathrm{ng} / \mathrm{mL})$ according to pharmacokinetic and simulated virtual data [13].

A recent study by Samama et al. [14] tested the effect of rivaroxaban on multiple, commercially available hemostasis assays, including the CoaguChek XS ${ }^{\circledR}$ point-ofcare device, which uses a thromboplastin reagent with sensitivity comparable to Neoplastin ${ }^{\circledR}$ and TriniClot ${ }^{\circledR}$. In the study, rivaroxaban induced a concentration-dependent PT prolongation. This effect was linear over a broad concentration range with all PT reagents. The correlation between the PT ratio and the concentration of rivaroxaban determined by CoaguChek XS ${ }^{\circledR}$ was $R 2=0.997$ [14].

In our case, the patient reportedly last administered rivaroxaban $20 \mathrm{mg}$ in the morning. The INR was assessed 7-8 h later, approximately $4-5 \mathrm{~h}$ after the peak concentration, and the subsequent peak effect on PT. Using data from Kubitza et al. [11] we can estimate the PT prolongation in our case to be less than 4.4-fold (effect with maximum rivaroxaban concentrations) but greater than 1.6fold (effect with rivaroxaban monotherapy). His estimated creatinine clearance was $46 \mathrm{~mL} / \mathrm{min}$, suggesting an increased plasma concentration (1.5-fold) [9] vs. patients with normal renal function. His weight was $133 \mathrm{~kg}$, which has not been associated with relevant pharmacokinetic alterations [10]. Because we were unable to obtain the actual venous INR value or rivaroxaban plasma concentration, our ability for further analysis is limited.

\section{Improvement in Processes of Care}

Discovery of the inadvertent duplication of anticoagulant therapy in this patient prompted the staff to examine the case to identify and learn about any specific safety lapses. Such an analysis is useful to facilitate improvements within the healthcare delivery system and ultimately prevent medication errors and ADEs. In 2012, warfarin was the second most frequent medication reported directly to the US Food and Drug Administration as causing a safety issue, primarily hemorrhage [15]. The most frequent medication reported was the NOAC dabigatran, further highlighting the inherent risks associated with anticoagulant therapy. In our case, an ADE was averted and any bleeding consequence a near miss. The case also brings to attention how different elements of the system, including equipment and communication, have a role in the occurrence of a medication error (Fig. 1).

In this case, the anticoagulation staff contacted the patient's community pharmacy by telephone, placed the rivaroxaban order, and determined the patient's co-payment to be US $\$ 40$ per month. The prescription was then placed on hold in the patient's prescription profile, despite a request by the anticoagulation staff to cancel or discontinue it. Although it may be more labor intensive, a preferred strategy for determining medication cost is to contact the patient's specific insurance company rather than submitting a prescription claim at the retail pharmacy. In the USA, the medication co-payment for rivaroxaban may vary among users depending on the individual's insurance plan pharmacy benefit or lack thereof. The negotiated price of a prescription medication with the pharmaceutical industry may also differ in countries outside the USA and change the cost to the consumer. Cost is one factor that may affect the selection of a specific anticoagulation agent by physician and patient.

This case also highlights how the electronic medical record at the clinic site does not transmit discontinued medication orders to the dispensing pharmacy. Unless the prescribing provider or staff directly contacts the pharmacy to cancel or discontinue the prescription, the medication remains active, creating the opportunity for refill errors. This type of error can be alarmingly high. In a recent retrospective cohort study, it was found that among 83,902 medications electronically discontinued by a physician during a 12-month study period, 1218 were subsequently dispensed by the pharmacy [16]. Until the electronic medical record interface allows discontinued orders to be electronically transmitted to the dispensing pharmacy, it is critical to directly communicate this action to the pharmacy. In addition, prescribing providers and staff should be educated about the potential deficiency of the system to 
Fig. 1 Analysis of the medication error using an Ishikawa cause-and-effect diagram

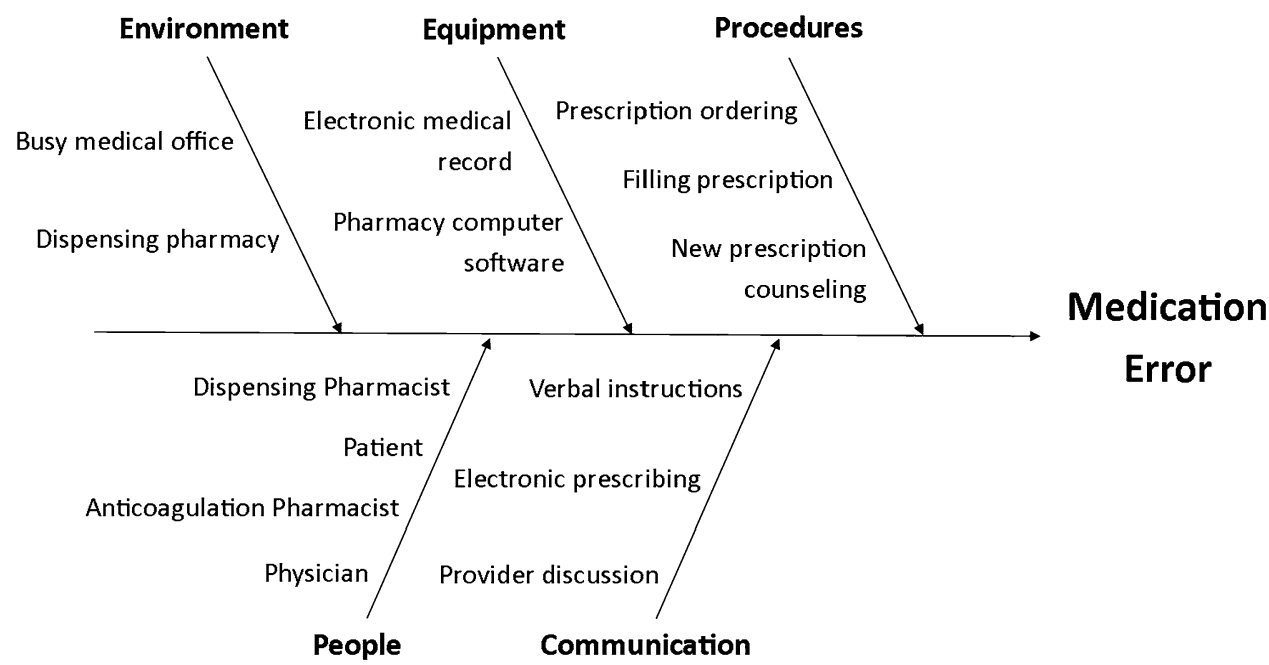

help optimize safe medication therapy. After performing our internal analysis, we updated our clinic procedures to recommend cost inquiries are made through the insurance company and all anticoagulant discontinuations are directly communicated to the dispensing pharmacy.

Quality communication at the patient level is also essential for risk reduction. Patients should be educated and encouraged to become more engaged with their healthcare management, including asking any questions to understand the intended use of all medications. A recent study showed how reviewing enhanced medication plans with patients upon hospital discharge improved patient knowledge of their individual medication treatment without prolonging the overall process [17]. Although this study was conducted in the inpatient setting, it highlights how process improvements can improve the quality of care and patient satisfaction without requiring additional time. Patients prescribed warfarin should continue to receive a handout listing medications to avoid at the initial visit, and this handout must be periodically reviewed as new agents are introduced into the market, such as NOACs. In this case, staff instructed the patient to continue warfarin and not to start rivaroxaban; however, a communication failure was experienced. Thorough documentation of any patient encounter is necessary to reduce the risk of medical liability. In this case, documentation of actual patient understanding to avoid concurrent use of rivaroxaban and warfarin was completed only after discovery of the medication error.

Poor communication during the prescription entry and verification processes also increased the likelihood of this medication error. Performing a thorough medication use review at the dispensing pharmacy site is an essential step to promote safe medication therapy. In this case, the active medication list included both warfarin and rivaroxaban, dispensed only 8 days apart, and this should have prompted an inquiry to the patient or anticoagulation clinic. If a patient uses multiple pharmacies, the opportunity for intervention would be less likely, and this highlights the importance of encouraging patients to maintain only one dispensing pharmacy. In the future, additional caution on behalf of the dispensing pharmacist is warranted given the possibility of serious harm. Patient counseling can be an effective strategy to reduce medication errors and is mandatory by law with every new prescription.

\section{Conclusion}

Sixty years after being approved for medical use, warfarin is still commonly used in clinical practice. NOACs offer many advantages compared with warfarin, yet possess sufficient disadvantages to leave a role for warfarin therapy moving forward. As anticoagulant therapy continues to evolve, the potential for medication errors will increase as clinical practice adapts to emerging treatment options. Given the high-risk nature of these medications, it is imperative to identify and prevent errors in the prescribing, transcription, and dispensing processes. Anticoagulation clinic staff must be knowledgeable and prepared to help manage the unique situations and challenges created by NOACs. In this case report, the authors describe inadvertent duplication of warfarin and rivaroxaban, and the subsequent challenges with interpreting the coagulation assay and determining clinical management. We attempted to apply available pharmacokinetic and laboratory studies to help guide therapy. Analysis after the event revealed several strategies to decrease the likelihood of recurrence. The potential safety issues and uncertainty that inherently come with using NOACs can be improved with communication among healthcare professionals. It is important to report 
such errors, through case reports and voluntary submission to the Food and Drug Administration, to improve patient safety.

Acknowledgments Julie Fusco, Eric Paulus, Alexandra Shubat, and Sharminara Miah declare they have no conflict of interest. No financial support was received for the preparation of this manuscript.

Funding The authors received no sources of funding that require acknowledgment.

Consent Written informed consent was obtained from the patient for publication of this case report. A copy of the written consent is available for review by the Editor-in-Chief of this journal.

Open Access This article is distributed under the terms of the Creative Commons Attribution-NonCommercial 4.0 International License (http://creativecommons.org/licenses/by-nc/4.0/), which permits any noncommercial use, distribution, and reproduction in any medium, provided you give appropriate credit to the original author(s) and the source, provide a link to the Creative Commons license, and indicate if changes were made.

\section{References}

1. Raskob GE, Silverstein R, Bratzler DW, et al. Surveillance for deep vein thrombosis and pulmonary embolism: recommendations from a national workshop. Am J Prev Med. 2010;38(4 Suppl):S502-9.

2. Kitslaar DB, Wysokinski WE, McBane RD 2nd. The role of novel anticoagulants in the management of venous thromboembolic disease. Curr Treat Options Cardiovasc Med. 2014;16(8):326.

3. Scaglione F. New oral anticoagulants: comparative pharmacology with vitamin $\mathrm{k}$ antagonists. Clin Pharmacokinet. 2013;52(2):69-82.

4. Budnitz DS, Shehab N, Kegler SR, et al. Medication use leading to emergency department visits for adverse drug events in older adults. Ann Intern Med. 2007;147(11):755-65.

5. Sigrid N, Solhaug V, Myhr K, et al. Warfarin associated bleeding events and concomitant use of potentially interacting medicines reported to Norwegian spontaneous reporting system. Br J Clin Pharmacol. 2011;71(2):254-62.
6. Xarelto ${ }^{\circledR}$ (package insert). Titusville, NJ. Janssen Pharmaceuticals Inc. 2011.

7. Kubitza D, Becka M, Mueck W, et al. Safety, tolerability, pharmacodynamics, and pharmacokinetics of rivaroxaban, an oral, direct factor Xa inhibitor, are not affected by aspirin. J Clin Pharmacol. 2006;46(9):981-90.

8. Graff J, von Hentig N, Misselwitz F, et al. Effects of the oral, direct factor $\mathrm{Xa}$ inhibitor rivaroxaban on platelet-induced thrombin generation and prothrombinase activity. J Clin Pharmacol. 2007;47(11):1398-407.

9. Kubitza D, Becka M, Mueck W, et al. Effects of renal impairment on the pharmacokinetics, pharmacodynamics and safety of rivaroxaban, an oral, direct Factor Xa inhibitor. Br J Clin Pharmacol. 2010;70(5):703-12.

10. Kubitza D, Becka M, Zuehlsdorf M, et al. Body weight has limited influence of the safety, tolerability, pharmacokinetics, or pharmacodynamics of rivaroxaban in healthy subjects. J Clin Pharmacol. 2007;47(2):218-26.

11. Kubitza D, Becka M, Mueck W, et al. Pharmacodynamics and pharmacokinetics during the transition from warfarin to rivaroxaban: a randomized study in healthy subjects. Br J Clin Pharmacol. 2014;78(2):353-63.

12. Meuck W, Borris LC, Dahl OE, et al. Population pharmacokinetics and pharmacodynamics of once- and twice-daily rivaroxaban for the prevention of venous thromboembolism in patients undergoing total hip replacement. Thromb Haemost. 2008;100(3):453-61.

13. Mueck W, Lensing AW, Agnelli G, et al. Rivaroxaban: population pharmacokinetic analyses in patients treated for acute deepvein thrombosis and exposure simulations in patients with atrial fibrillation treated for stroke prevention. Clin Pharmacokinet. 2011;50(10):675-86.

14. Samama MM, Martinoli JL, LeFlem L, et al. Assessment of laboratory assays to measure rivaroxaban: an oral, direct factor Xa inhibitor. Thromb Haemost. 2010;103(4):815-25.

15. QuarterWatch. Leading Drug Safety Issues of 2012 [Institute for Safe Medical Practices web site]. October 17, 2013. Available at: http://www.ismp.org/quarterwatch/pdfs/2012Q4.pdf. Accessed 15 Mar 2015.

16. Allen AS, Sequist TD. Pharmacy dispensing of electronically discontinued medications. Ann Intern Med. 2012;157(10):700-5.

17. Send AF, Schwab M, Gauss A, et al. Pilot study to assess the influence of an enhanced medication plan on patient knowledge at hospital discharge. Eur J Clin Pharmacol. 2014;70(10):1243-50. 